\title{
Surface treatment by the ion flow from electron beam generated plasma in the forevacuum pressure range
}

\author{
Aleksandr Klimov ${ }^{1, *}$, and Aleksey Zenin ${ }^{1}$ \\ ${ }^{1}$ Tomsk State University of Control Systems and Radioelectronics, 634050 Tomsk, Russia
}

\begin{abstract}
The paper presents research results of peculiarities of gas ion flows usage and their generation from large plasma formation $(>50 \mathrm{sq} . \mathrm{cm})$ obtained by electron beam ionization of gas in the forevacuum pressure range. An upgraded source was used for electron beam generation, which allowed obtaining ribbon electron beam with no transmitting magnetic field. Absence of magnetic field in the area of ion flow formation enables to obtain directed ion flows without distorting their trajectories. In this case, independent control of current and ion energy is possible. The influence of electron beam parameters on the parameters of beam plasma and ion flow - current energy and density - was determined. The results of alumina ceramics treatment with a beam plasma ions flow are given.
\end{abstract}

\section{Introduction}

Electron-beam plasma generated while electron beam is transmitted through gaseous atmosphere of a vacuum chamber with the energy of several $\mathrm{keV}$ [1] is widely used in various material treatment technologies [2-5]. The parameters of beam plasma can be controlled in a wide range, unlike those of various types of discharge-generated plasma. Variation of electron beam current and energy, as well as composition of gaseous atmosphere, enables variation of plasma density and temperature. Due to low temperature of electron component and relatively high concentration beam plasma is used for etching of thin (monoatomic) surface layer [6-7].

Plasma formation with the electron temperature higher than several tens of $\mathrm{eV}$ can be obtained by providing conditions for combined interaction of electron beam and plasma, i.e. beam plasma instability [8]. Because of such instability beam electrons transmit part of their energy to Langmuir waves which while damping transmit their energy to thermal plasma electrons [9]. As a result, chemical activity of plasma increases, and such plasma can be effectively used in plasma-chemical technologies [10].

It should be noted that the optimum pressure range for generating dense beam plasma is between 1 and $100 \mathrm{~Pa}$ [11]. High-voltage glow discharge (HVGD) electron sources are more often used for this pressure range [12]. However, while using such sources there is almost no possibility for independent control of electron beam current and energy. Electron

Corresponding author: klimov@main.tusur.ru 
sources with thermionic cathode which generate beams in the pressure range between 0.001 and $0.1 \mathrm{~Pa}$ can be used to control beam parameters. But in this case differential pressure must be provided between the electron gun chamber and the chamber where beam plasma is generated [13]. This condition, in the end, makes the technical unit more complex.

The works $[14,15]$ describe effective usage of plasma cathode electron sources for generating large-area beam plasma. Control of energy spectrum of plasma ion flow in the mentioned works was performed by putting negative bias on the target. Still, it should be noted that this method cannot be used for non-conductive materials treatment, for no potential can be applied in this case. The problem of continuous electron beams generation in the pressure range between 5 and $100 \mathrm{~Pa}$ is solved by using the so-called forevacuum plasma electron sources [16]. Such sources use hollow cathode discharge to generate dense discharge plasma. The apparatus also contains accelerating space which prevents from disruption at high pressure values.

During transmission of a ribbon electron beam through gaseous medium of a vacuum chamber in forevacuum pressure range a large-area plasma formation is generated (more than 0.5 sq.m), which is called a plasma sheet [17]. Special structure of accelerating space [18] enables to obtain electron beam without traditionally used transmitting magnetic field. As described in [18, 19], plasma ion flows can compensate for the negative charge transmitted to the target in the process of electron beam treatment. It is obvious that such compensation is possible in case of plasma ion flow treatment due to higher mobility of plasma electron component. At the same time, absence of magnetic field allows electrons to fall on the target without their trajectory distortion. The paper presents investigation results on the influence of electron beam parameters on concentration of the generated beam plasma, as well as on the parameters of plasma ion flows. Plasma in this case is generated when ribbon electron beam is transmitted through gaseous medium at the pressure of $10 \mathrm{~Pa}$. Novelty of the research is based upon absence of magnetic field in the beam transmission region. The paper also gives the results of using beam plasma for ceramic material surface treatment, particularly polycor material.

\section{Experiment}

Experimental stusy was performed using the vacuum unit consisting of a rectangular vacuum chamber sized $0.4 \times 0.7 \times 0.4 \mathrm{~m}$ with mechanical system of air pumping, a ribbon electron beam source and a power supply and diagnostic system. Pressure in the vacuum chamber was kept on the level of 5-10 Pa with the help of BocEdwards 80 forevacuum pump and was controlled by the inlet valve. The ribbon electron beam was produced by a forevacuum plasma electron source [18]. The source was specially designed for ribbon beam generating while transmission without traditionally used [15, 19, 20] longitudinal magnetic field. The source represented a three-electrode system - hollow cathode 1 , flat anode 2 and extractor 3 (Fig. 1). Structure and operation scheme of the source are described in more detail in [18]. 


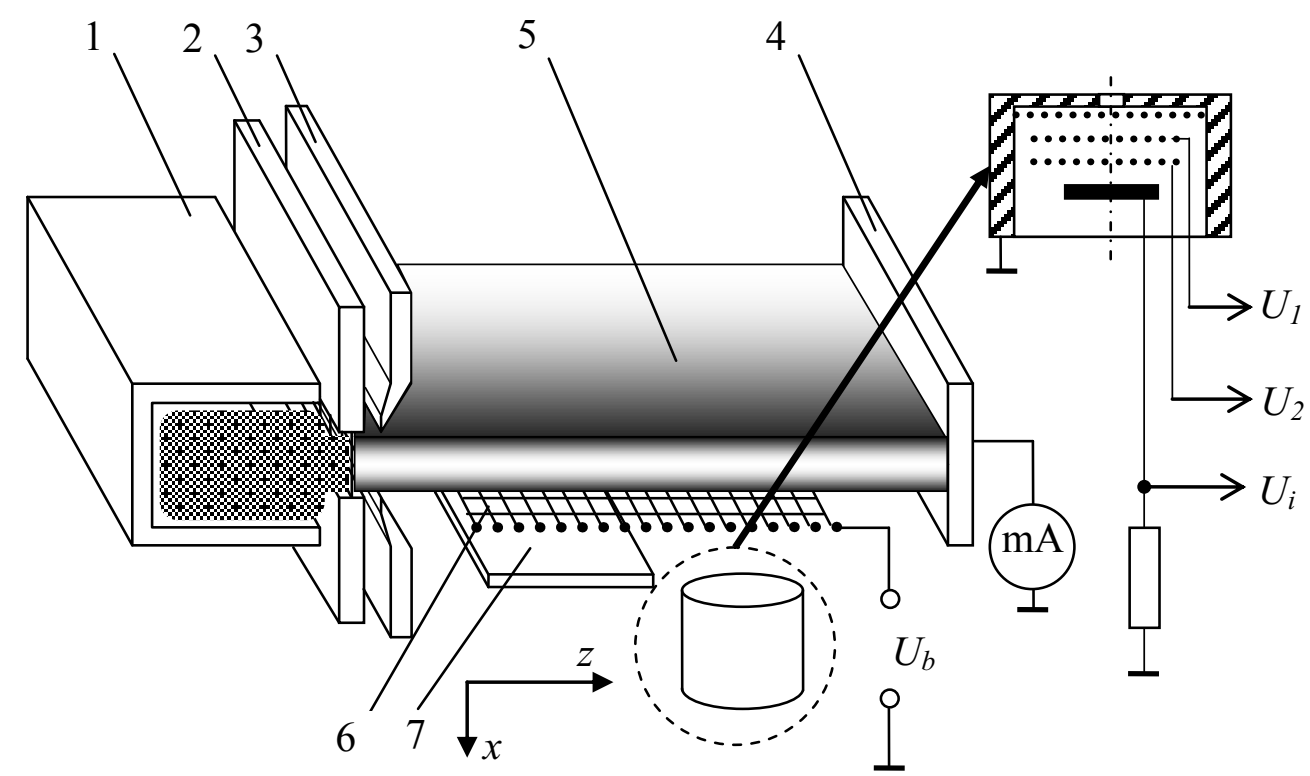

Fig. 1. Scheme of the experiment and the energy analyzer.

After transition in the vacuum chamber the electron beam generated by plasma source is caught by a collector 4 . While making their way to the collector beam electrons were performing ionization of gas molecules and generated beam plasma, the area of which was defined by the beam parameters. A single probe in the form of copper wire $0.3 \mathrm{~mm}$ in diameter placed in a ceramic tube was used to measure plasma parameters. $5 \mathrm{~mm}$ of the probe receiver part was extruded from the tube. Plasma concentration was evaluated on the basis of probe characteristics using standard method [21]. To extract ions from beam plasma and create clear plasma edge a flat extraction grid 6 was used placed parallel to the beam at the distance of $2 \mathrm{~cm}$ from its mean plane. Input of negative bias $U_{b} 10-50 \mathrm{~V}$ to the grid enabled to extract ions with controlled energy. Targets 7 represented by ceramic plates made of polycor with thickness of $1 \mathrm{~mm}$ and size of $10 \times 10 \mathrm{~mm}^{2}$ were placed $1 \mathrm{~cm}$ behind the grid. Energy spectrum of ions hitting the target was investigated using a multigrid energy analyzer (Fig. 1). It was positioned behind the grid in the way that its axis is perpendicular to the beam propagation plane. Screening grid was placed behind the analyzer inlet with the diameter of $5 \mathrm{~mm}$ and depth of $2 \mathrm{~mm}$ and was electrically connected with the energy analyzer body which was grounded. Retarding potential U1 in the range between 0 and $100 \mathrm{~V}$ was supplied to the second grid. To eliminate the effect of secondary electron emission on the collector placed behind the second grid, negative (in relation to the analyzer body) potential $\mathrm{U}_{2}$ equal to $10 \mathrm{~V}$ was supplied to the second grid. Induced retarding field prevented secondary electrons from scattering from the collector. The grids were made from stainless steel and had the cell size of $0.3 \times 0.3 \mathrm{~mm}$. The distance between the grids was $2 \mathrm{~mm}$. Current signal from the collector was transmitted to a resistor of 10 $\mathrm{kOhm}$ and then registered by Tektronix 2024B digital storage oscilloscope. Ions energy spectrum was calculated by derivation of the curve of ion current delay. 


\section{Results}

The research results showed that beam plasma concentration monotonically decreases while electron beam is transmitted from vacuum source to collector in a vacuum chamber (Fig. 2), as well as while distancing from the mean plane of the beam (Fig. 3). According to the authors, the most probable reason for such concentration behavior is electron beam expansion due to scattering on residual gas molecules. Increase of accelerating voltage allows producing a beam with lower divergence; however, in this case the beam plasma region is moving towards the mean plane of the beam and drops to the periphery. Besides, from the point of view of effective interaction between beam electrons and residual atmosphere atoms and molecules, the use of low-energy electron beam is more advisable given the fact that ionization cross section corresponds to the energy regions of tens and hundreds $\mathrm{eV}[22]$.

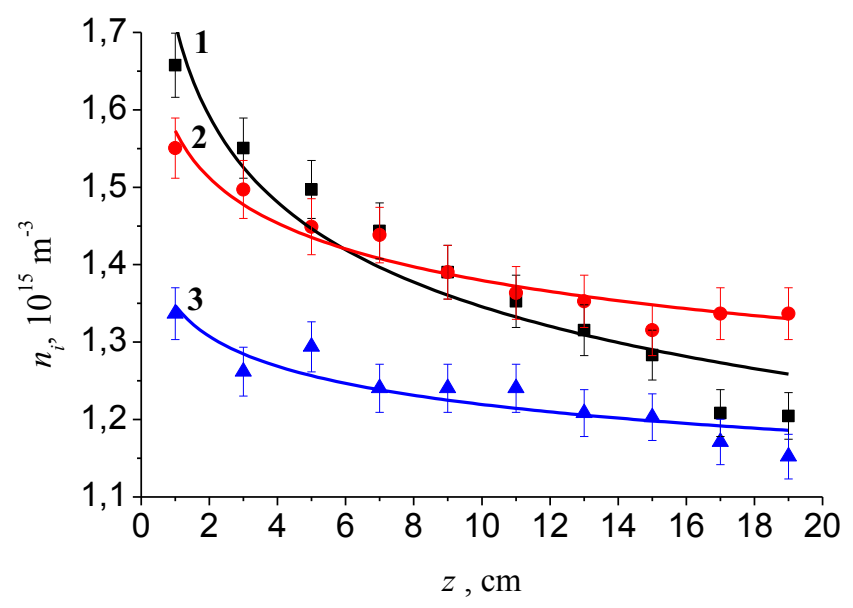

Fig. 2. Dependence of beam plasma concentration $n$ on transmission area $z$ for various distances from mean plane of the beam; distance to the mean plane of the $x$ beam: $1-1 \mathrm{~cm} ; 2-3 \mathrm{~cm} ; 3-5 \mathrm{~cm}$. Accelerating voltage $U_{a}=3 \mathrm{kV}$ and pressure $p=10 \mathrm{~Pa}$.

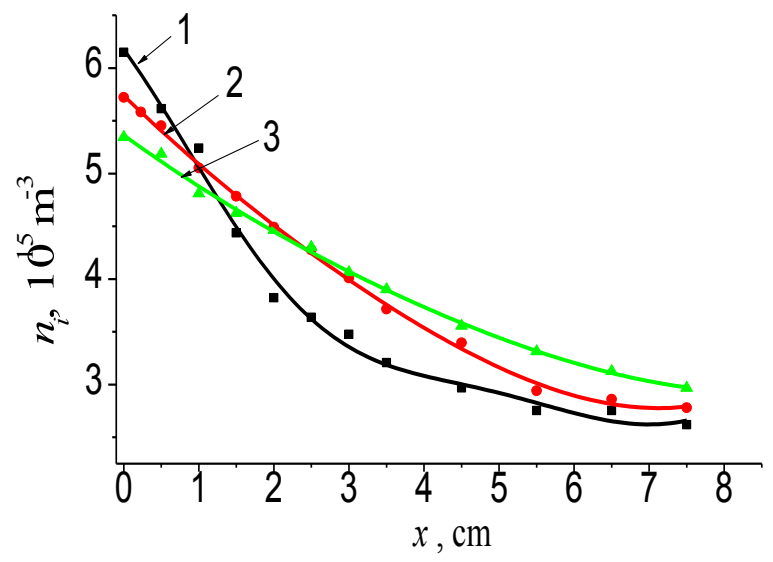

Fig. 3. Beam plasma concentration $n$ depending on the distance to the mean plane of the beam $r$ for various lengths of transmission areas $z: 1-1 \mathrm{~cm} ; 2-10 \mathrm{~cm} ; 3-20 \mathrm{~cm}$. Accelerating voltage $U_{a}=3$ $\mathrm{kV}$ and pressure $p=10 \mathrm{~Pa}$. 
Decrease of beam plasma concentration in the measurement point with the increase of accelerating voltage is indirectly proved by the dependence of ion current to the collector placed behind the extraction grid 6 (Fig. 1). As can be seen from Figure 4, increase of beam electrons energy leads to decrease of registered ion current; and due to the fact that the collector is placed at a fixed distance from the mean plane of the beam decrease of plasma concentration in proximity to the extraction grid causes decrease of ion current density on collector.

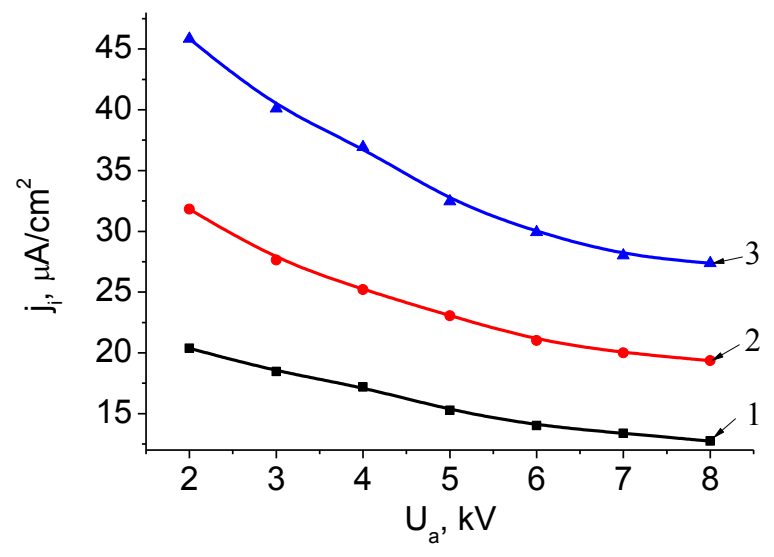

Fig. 4. Dependence of ion current density $j_{i}$ on accelerating voltage $U_{a}$ for various beam current values $I_{b}: 1-180 \mathrm{~mA}, 2-360 \mathrm{~mA}, 3-550 \mathrm{~mA}$. Pressure equals to $8 \mathrm{~Pa}$.

Dependence of ion current density on the distance to extraction grid presented in Figure 5 enables to evaluate ion loss when propagating in forevacuum pressure region and to determine the extent of ion current control in the process of further surface treatment. As expected, the highest value of ion current density is observed in proximity to extraction grid. Current density decreases while distancing from the grid plane.

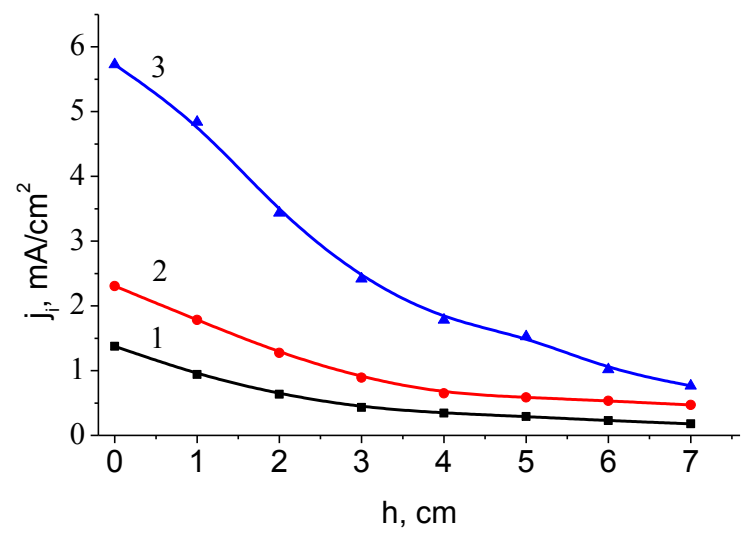

Fig. 5. Dependence of ion current density $j_{i}$ on the distance to mean plane of the beam $h$ for various beam current values $I_{b}: 1-180 \mathrm{~mA}, 2-360 \mathrm{~mA}, 3-550 \mathrm{~mA}$. Pressure equals to $8 \mathrm{~Pa}$.

Another means to control ion current parameters is ion energy control. The experiments showed that ion flow energy is primarily determined by the extraction grid potential and plasma potential. Within the experiments, negative voltage (in relation to grounded 
chamber walls) in the range of -10 to $-50 \mathrm{~V}$ was supplied to the extraction grid. Figure 6 shows ion energy distribution depending on the potential supplied to the extraction grid. The peak of energy spectrum is higher than the grid potential by the value of about $10 \mathrm{eV}$, which can be related to the positive potential of beam plasma. This means that negative potential supplied to the grid causes additional acceleration of ions that approach plasma edge.

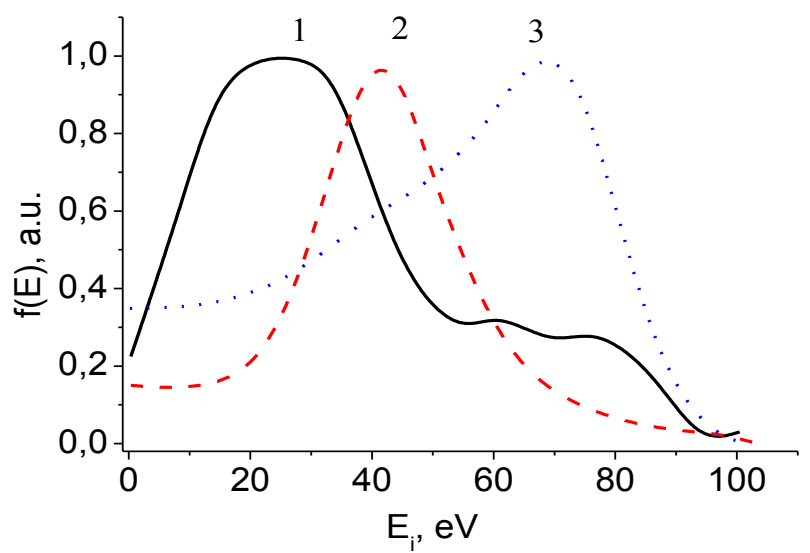

Fig. 6. Normalized functions of ion energies distribution $f\left(E_{i}\right)$ depending on voltage on the extraction grid $\mathrm{U}_{\mathrm{b}}: 1-10 \mathrm{~V}, 2-30 \mathrm{~V}, 3-60 \mathrm{~V}$.

Plates made from polycor were treated using the ion flow extracted from beam plasma. Ceramic plates were placed at the distance of $1 \mathrm{~cm}$ from the extraction grid, ion beam current density was $50 \mu \mathrm{A} / \mathrm{cm}^{2}$. The plates were treated for 30 minutes. As a result, the surface became rougher compared to the initial material (Fig. 7).
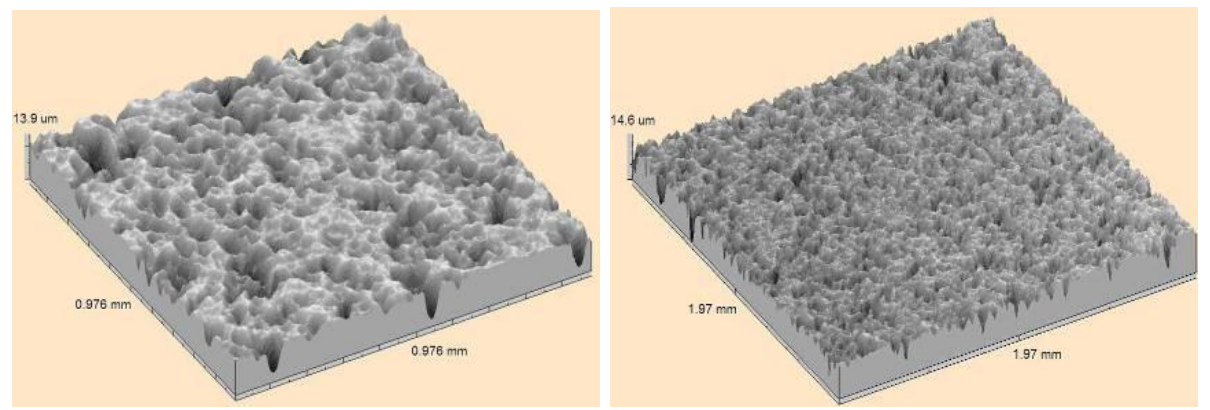

Fig. 7. Ceramic surface before (on the left) and after (on the right) ion beam treatment.

The fact of variation in surface morphology enables to talk about using a forevacuum plasma electron source for beam plasma generation and its application for treatment of long non-conductive products.

\section{Conclusion}

The research shows that the use of plasma electron source able to operate in forevacuum pressure regions allows generating large-area beam plasma. Absence of magnetic field in transmission area causes only minor electron beam expansion. Density of beam plasma ion 
flow is defined by the beam current, accelerating voltage and distance of the beam transmission. While using extraction grid with set potential it is possible to obtain ion flows with controlled energy, which is especially important for treatment of various surfaces. Ion treatment of non-conductive materials surfaces (polycor) leads to variation in their morphology, particularly increases their roughness. This can be used for obtaining durable materials with extended surface.

Further research in this field can be focused on increasing ion flow density. This can be implemented by initiating beam plasma discharge while electron beam transmission through gas.

The work was supported by the Ministry of Education and Science of the Russian Federation, grant No. 3.9605.2017/8.9.

\section{References}

1. J.J. Rocca, J.D. Meyer, M.R. Farrell, G.J. Collins, J. Appl. Phys., 56, 790 (1984)

2. D.R. Borisa, T.B. Petrova, G.M. Petrov, S.G. Walton, J. Vac. Sci. Technol., A 35, $01 \mathrm{~A} 104$ (2017)

3. S. Walton, D. Leonhardt, D. Murphy, R. Meger, R. Fernsler, Bull. Am. Phys. Soc., 44, 8, 58 (1999)

4. D. Leonhardt, C. Muratore, S.G. Walton, IEEE Trans. on Plasma Science, 33, 2, 783 (2005)

5. T.M. Vasilieva, Journal of Physics: Conference Series, 370, 012012 (2012)

6. A. Agarwal, M.J. Kushner, J. Vac. Sci. Technol., A 27, 37 (2009)

7. S.G. Walton, D.R. Boris, S.C. Hernández, E.H. Lock, Tz.B. Petrova, G.M. Petrov, R.F. Fernsler, J. Solid State Sci. Technol., 4(6), 5033-5040 (2015)

8. I. Alexeff, R.V. Neidigh, et all., Phys. Rev. Letters, 10(7), 273-276 (1963)

9. W.D. Getty, L.D. Smullin, J. Appl. Phys., 34, 3421 (1963)

10. E.G. Shustin, N.V. Isaev, M.P. Temiryazeva, Yu.V. Fedorov, Vacuum 83, 1350-1354 (2009)

11. R.F. Fernsler, W.M. Manheimer, R.A. Meger, J. Mathew, D.P. Murphy, R.E. Pechacek, J.A. Gregorc, Physics of Plasmas, 5(5), 2137 (1998)

12. I.V. Melnik, Radioelectronics and communication systems, 56(12), 51 - 61 (2013)

13. G.P. Berezina, V.S. Us, Plasma Physics Reports, 37(13), 1156-1161 (2011)

14. W.M. Manheimer, R.F. Fernsler, M. Lampe, R.A. Meger, Plasma Sources Sci. Technol., 9, 370 (2000)

15. S.G. Walton, C. Muratore, D. Leonhardt, R.F. Fernsler, D.D. Blackwell, R.A. Meger, Surf. Coat. Technol., 186(1-2), 40 (2004)

16. V.A. Burdovitsin, Y.A. Burachevskii, E.M. Oks, M.V. Fedorov, Instrum. Exp. Tech., 46, 257 (2003)

17. V.A. Burdovitsin, E.M. Oks, M.V. Fedorov, Russ. Phys. J., 47, 310-314 (2004)

18. A.S. Klimov, M.I. Lomaev, E.M. Oks, A.P. Andreichik, Tech. Phys., 62, 218 (2017)

19. R.A. Meger et al., Physics of plasmas, 8(5), 2558 (2001)

20. E.G. Shustin, N.V. Isaev, I.L. Klykov, V.V. Peskov, Vacuum, 85, 711 (2011)

21. F.F. Chen, Electric Probes, in Plasma Diagnostic Techniques, Chapter 4, 113-200 (Academic Press, New York, 1965)

22. Y. Nakamura, M. J. Kurachi, Phys. D, 21(5), 718 (1988) 